\title{
Haloperidol Selectively Remodels Striatal Indirect Pathway Circuits
}

\author{
Luke E Sebel', Steven M Graves', C Savio Chan' and D James Surmeier, \\ 'Department of Physiology, Feinberg School of Medicine, Northwestern University, Chicago, IL, USA
}

Typical antipsychotic drugs are widely thought to alleviate the positive symptoms of schizophrenia by antagonizing dopamine $D_{2}$ receptors expressed by striatal spiny projection neurons (SPNs). What is less clear is why antipsychotics have a therapeutic latency of weeks. Using a combination of physiological and anatomical approaches in ex vivo brain slices from transgenic mice, it was found that 2 weeks of haloperidol treatment induced both intrinsic and synaptic adaptations specifically within indirect pathway SPNs (iSPNs). Perphenazine treatment had similar effects. Some of these adaptations were homeostatic, including a drop in intrinsic excitability and pruning of excitatory corticostriatal glutamatergic synapses. However, haloperidol treatment also led to strengthening of a subset of excitatory corticostriatal synapses. This slow remodeling of corticostriatal iSPN circuitry is likely to play a role in mediating the delayed therapeutic action of neuroleptics.

Neuropsychopharmacology (2017) 42, 963-973; doi:10.1038/npp.2016.173; published online 12 October 2016

\section{INTRODUCTION}

Dopamine signaling in the basal ganglia assists in goaldirected action selection and habit formation by providing information about the outcome of goal-directed behavior and the motivational salience of stimuli (Redgrave et al, 2010; Winton-Brown et al, 2014). Dopamine fibers 'broadcast' this signal to all cell types within the striatum, but the major target of this innervation is the principal spiny projection neuron (SPN) (Gerfen and Surmeier, 2011). There are two main classes of SPNs. The so-called direct pathway SPNs (dSPNs) promote the selection of appropriate actions and indirect pathway SPNs (iSPNs) help suppress unwanted ones (Frank, 2011). Dopamine bidirectionally modulates the activity of these two classes of SPNs. This bidirectionality reflects the dichotomous expression of $\mathrm{G}$ protein-coupled DA receptors (GPCR) by these two SPN populations: dSPNs express $D_{1}$ receptors $\left(D_{1} R\right)$ that enhance excitability and excitatory glutamatergic synaptic transmission, whereas iSPNs express $D_{2}$ receptors $\left(D_{2} R s\right)$ that diminish excitability and excitatory synaptic transmission.

Antipsychotic drugs antagonize dopamine $\mathrm{D}_{2} \mathrm{Rs}$ at concentrations that correlate with their clinical potency (Creese et al, 1976; Seeman et al, 1976); however, their therapeutic benefits lag behind the acute blockade of $\mathrm{D}_{2} \mathrm{Rs}$ by days, weeks, or even months (Agid et al, 2003; Leucht et al, 2005; Tauscher et al, 2002). Slow alterations in corticostriatal

*Correspondence: Dr D James Surmeier, Department of Physiology, Feinberg School of Medicine, Northwestern University, 303 E Superior, Chicago, IL 606 I I, USA, Tel: + I 312503 4904, Fax: + I 312503 5 I01, E-mail: j-surmeier@northwestern.edu

Received 23 September 2015; revised 8 August 2016; accepted 10 August 2016; accepted article preview online 31 August 2016 circuitry might underlie the time lag between neuroleptic treatment and effect (Konradi and Heckers, 2001). Animal studies investigating the chronic effects of the typical antipsychotic haloperidol have consistently demonstrated ultrastructural alterations in the striatum, including an increase in the number of perforated synapses (Meshul and Casey, 1989; Meshul et al, 1996; Roberts et al, 1995). In line with the anatomical data, chronic haloperidol was shown to cause an alteration in the induction of striatal synaptic plasticity in acute brain slices from rodents, biasing synaptic plasticity toward the potentiation of glutamatergic inputs on striatal neurons (Centonze et al, 2004). This site of action is consistent with studies implicating striatal glutamatergic signaling in the pathophysiology of schizophrenia (Laruelle et al, 2003).

Despite the plausibility of this hypothesis, the effect of chronic antipsychotic treatment on striatal physiology has not been examined systematically. Moreover, the anatomical changes induced by antipsychotic drugs are controversial. For instance, chronic haloperidol has been reported to both increase (Kerns et al, 1992) and decrease (Kelley et al, 1997) spine density in the rat striatum. To reexamine the question with advanced tools, the effects of chronic haloperidol administration on the dendritic morphology and physiology of identified iSPNs and dSPNs were studied using a combination of optogenetic, two-photon laser scanning microscopy and electrophysiological approaches in ex vivo brain slices from mice. These studies show that haloperidol treatment induces a slow remodeling of the corticostriatal indirect pathway implicated in the positive symptoms of schizophrenia. 


\section{MATERIALS AND METHODS}

\section{Haloperidol Treatment}

Male and female hemizygous bacterial artificial chromosome
(BAC) transgenic mice (p28-p38) expressing eGFP under either Drd1a or Drd2 control (Chan et al, 2012) were treated with intraperitoneally (IP) injected haloperidol-HCl (1 mg/kg) (Tocris Bristol, UK), perphenazine (4 mg/kg)

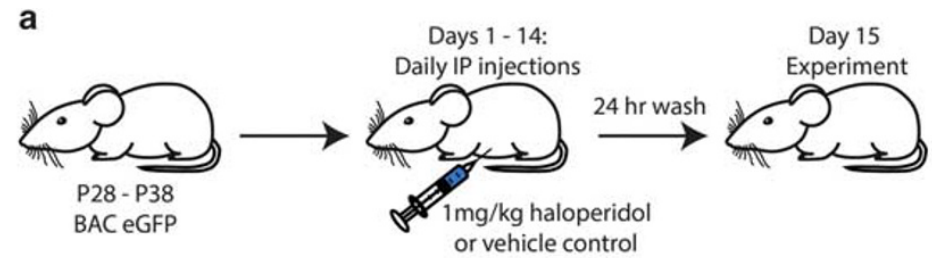

b

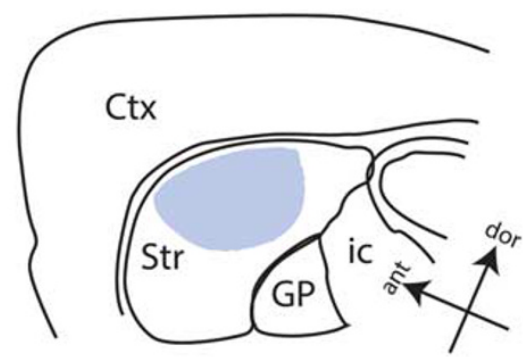

d

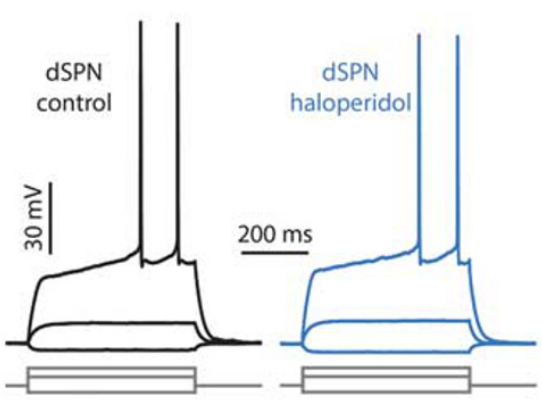

f
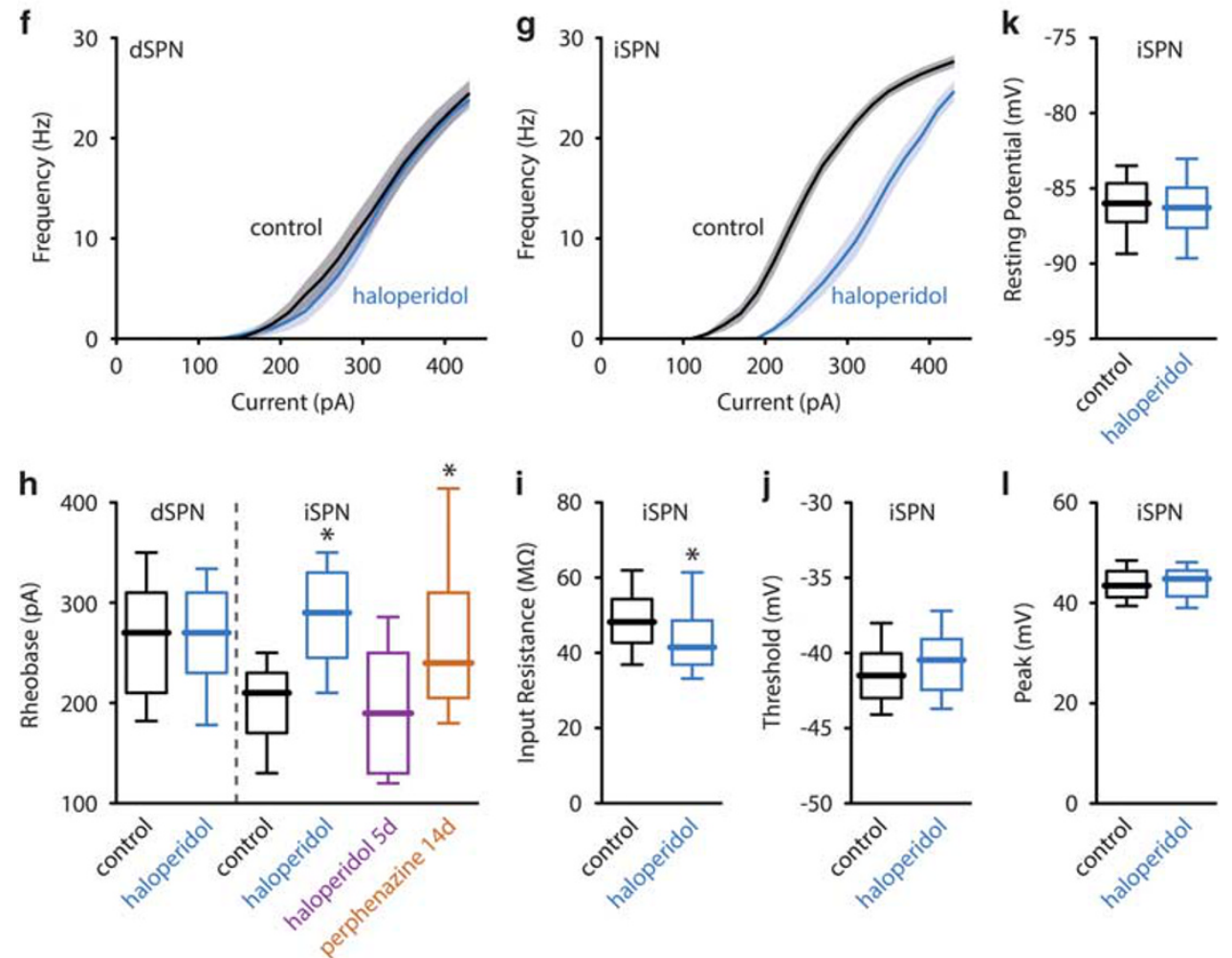

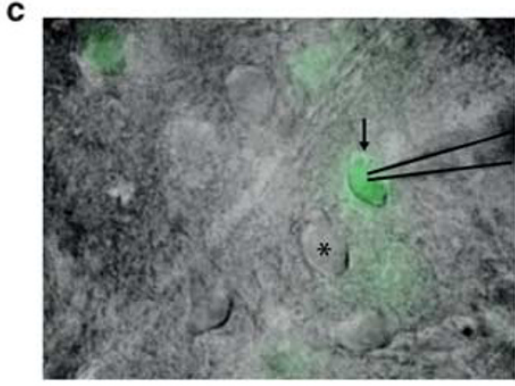

e

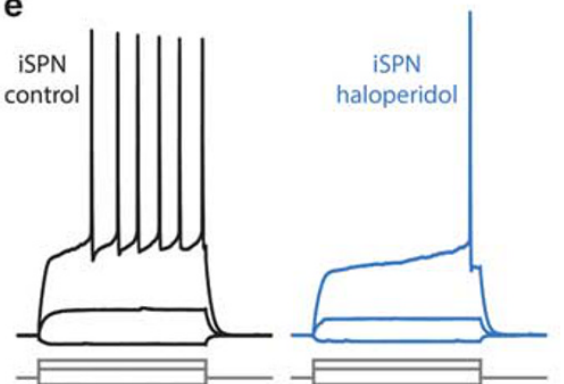

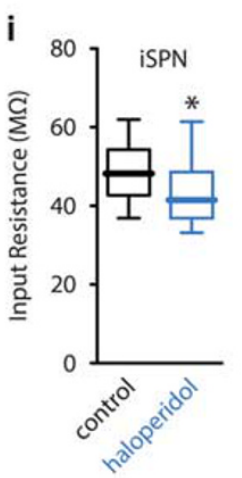
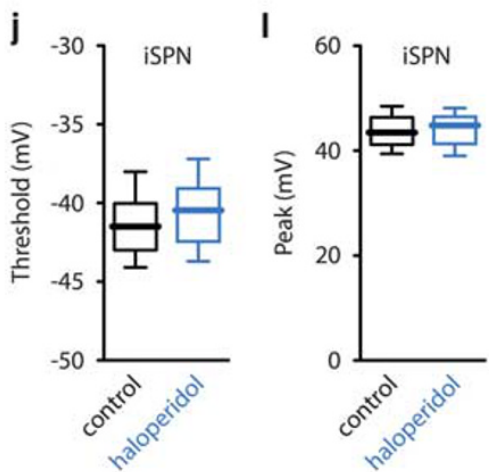
(Sigma-Aldrich, St Louis, MO), or $0.9 \%$ saline control for 14 days, except where otherwise noted (Figure 1a). We began injecting at postnatal week 5 to ensure adequate striatal development (Tepper et al, 1998). Injections were halted $24 \mathrm{~h}$ before experiments to ensure complete drug washout (Kirschbaum et al, 2008). In a subset of experiments, animals were injected with a single dose of haloperidol or 5 days of haloperidol before a $24 \mathrm{~h}$ washout before experimentation. A final subset of experiments used primed mini-osmotic pumps (Alzet, Cupertino, CA) to continuously deliver $0.25 \mathrm{mg} / \mathrm{kg}$ per day of haloperidol-HCl or vehicle control for 14 days, as previously described (Ilijic et al, 2011). Pumps were removed $24 \mathrm{~h}$ before experimentation. For circuit mapping experiments, $\operatorname{Drd} 2$ eGFP mice were crossed with a transgenic line that expresses channelrhodopsin-2 in the cortex (Thy1-ChR2) (Fieblinger et al, 2014). All mice were housed on a $12 \mathrm{~h}$ light/dark schedule with food and water available ad libitum. All protocols described here were reviewed and approved by the Northwestern Institutional Animal Care and Use Committee and all efforts were made to minimize suffering.

\section{Brain Slice Preparation}

Following $24 \mathrm{~h}$ of washout (Figure 1a), mice were deeply anesthetized with a mixture of ketamine $(50 \mathrm{mg} / \mathrm{kg})$ and xylazine $(4.5 \mathrm{mg} / \mathrm{kg})$, and acute ex vivo brain slices were prepared as previously described (Chan et al, 2012) with the following modification: $240 \mu \mathrm{m}$ parasagittal slices were taken by blocking the brain midsagittally and gluing medial side down on a $\sim 15^{\circ}$ agar block to preserve corticostriatal projections for electrical stimulation.

\section{Whole-Cell Patch-Clamp Recording}

Slices were transferred to a submersion-style recording chamber on a BX51 upright, fixed-stage microscope (Olympus, Center Valley, PA) and continuously superfused with aCSF containing the following (in $\mathrm{mM}$ ): $125 \mathrm{NaCl}, 2.5$ $\mathrm{KCl}, 1.25 \mathrm{NaH}_{2} \mathrm{PO}_{4}, 25 \mathrm{NaHCO}_{3}, 1 \mathrm{MgCl}_{2}, 2 \mathrm{CaCl}_{2}$, and 13.9 $\mathrm{D}(+)$-Glucose. Slices were visualized under differential interference contrast (DIC) and somatic eGFP expression was checked using epifluorescence microscopy to verify cell identity (Figure 1c). Thick-walled (1.5 $\mathrm{mm}$ outer diameter) borosilicate pipettes (Sutter Instrument Company, Novato, CA) were pulled on a Flaming/Brown micropipette puller (Sutter) and were 3-4M $\mathrm{M}$ when filled with recording solution. Electrophysiological recordings were obtained with a MultiClamp $700 \mathrm{~A}$ amplifier interfaced with a PC running pClamp 10 (Molecular Devices, Sunnyvale, CA). Data were acquired at $50 \mathrm{kHz}$ and filtered at $10 \mathrm{kHz}$ except for $\mathrm{Sr}^{2+}$ miniature excitatory postsynaptic potentials (mEPSCs) that were filtered at $1 \mathrm{kHz}$, using an 8-pole Bessel filter and digitized using a DigiData 1322A A/D converter (Molecular Devices). The internal solution for current clamp recordings contained (in mM): $135 \mathrm{KMeSO}_{4}, 5 \mathrm{KCl}, 5$ HEPES, 5 EGTA, $0.5 \mathrm{CaCl}_{2}, 10 \mathrm{Na}_{2}$-phosphocreatine, $2 \mathrm{Mg}$-ATP, $0.5 \mathrm{Na}$-GTP, $\mathrm{pH}$ adjusted to 7.25-7.30 with $\mathrm{NaOH}, 300$ mOsm. The amplifier bridge circuit was adjusted to account for serial resistance and was continuously monitored. For voltage clamp recordings the pipettes were filled with a $\mathrm{Cs}^{+}$-based internal solution containing (in $\mathrm{mM}$ ): $130 \quad \mathrm{CsMeSO}_{3}$, 5 HEPES, 0.25 EGTA, $10 \mathrm{Na}_{2}$-phosphocreatine, $2 \mathrm{Mg}$-ATP, $0.5 \mathrm{Na}-\mathrm{GTP}, 5$ TEA-Cl (tetraethylammonium-Cl), 1 QX-314$\mathrm{Cl}, \mathrm{pH}$ adjusted to 7.25-7.30 with $\mathrm{CsOH}, 290$ mOsm. A $5 \mathrm{mV}$ test pulse was continually monitored during voltage clamp recordings and cells with an access resistance of over $20 \mathrm{M} \Omega$ or whose resistance changed $>20 \%$ were discarded. For experiments that employed cortical stimulation, a $200 \mu \mathrm{s}$ pulse was delivered via a platinum/iridium parallel bipolar electrode (FHC, Bowdoin, ME) placed between layers $\mathrm{V}$ and VI of the cortex.

To determine the AP threshold, phase plots of the first derivative of somatic membrane potential $(\mathrm{dV} / \mathrm{dt})$ were constructed and the inflection point of the membrane potential (where the slope reached $10 \mathrm{mV} / \mathrm{ms}$ ) was used as the threshold (Naundorf et al, 2006). Voltage-current plots were constructed from the equilibrium potentials reached for different somatic current injections ( -150 to $90 \mathrm{pA}, 20 \mathrm{pA}$ increments). $\mathrm{Sr}^{2+}$ mEPSCs were recorded as previously described (Ding et al, 2008).

\section{Subcellular Channelrhodopsin-Assisted Circuit Mapping (sCRACM)}

Slices were taken from haloperidol- or saline-treated Thy1ChR2-D2 mice and sCRACM was performed as previously described (Fieblinger et al, 2014) using standard aCSF supplemented with: $1.0 \mu \mathrm{M}$ TTX, $100 \mu \mathrm{M}$ 4-AP, $10 \mu \mathrm{M}$ SR-95531 hydrobromide (gabazine), and $1.0 \mu \mathrm{M}$ CGP 35348; $50 \mu \mathrm{M}$ Alexa Fluor 568 hydrazide sodium salt was added to the $\mathrm{Cs}^{+}$-based internal to allow visualization of spines.

Figure I Haloperidol decreased intrinsic excitability in iSPNs. (a) Example of haloperidol dosing schedule. (b) Schematic of a parasagittal $\left(\sim 15^{\circ}\right)$ slice depicting the cortex (Ctx), striatum (Str), globus pallidus (GP), and internal capsule (ic); the region in blue indicates the area of interest where cells were assayed. Arrows signify anterior (ant) and dorsal (dor) axis orientation. (c) $40 \times$ DIC image from a BAC D, eGFP animal with epifluorescence overlay (green) that shows a patch pipette (black lines) in contact with an iSPN (arrow) that is adjacent to a dSPN (asterisk). (d, e) Representative current-clamp recordings showing dSPNs and iSPNs treated with vehicle (black) or haloperidol (blue) for 14 days. The current injection protocol (diagram below) consisted of three 500 ms steps (-50, 90, and 290 pA). (f, g) dSPN intrinsic excitability was unchanged (control $n=25$, haloperidol $n=23$ ) and iSPN intrinsic excitability reduced (rightward shift in the current-response curve) by chronic haloperidol treatment (control $n=37$, haloperidol $n=34$ ). Shaded regions indicate SEM. ( $h$ ) Following I 4 days of haloperidol treatment, rheobase current did not change in dSPNs (control: 270 pA, $n=25$; haloperidol: 270 pA, $n=23$; Mann-Whitney test, $p=0.89)$. In iSPNs, 5 days of haloperidol did not change rheobase, but 14-day (14d) treatment with either haloperidol or perphenazine increased rheobase (control: 210 A,$n=37$; $5 \mathrm{~d}$ haloperidol: $190 \mathrm{pA}, n=16, p=0.99 ;$ I4d haloperidol: $290 \mathrm{pA}, n=34, p<0.000$ I; I4d perphenazine: 240 pA, $n=20$, $p=0.01$; Kruskal-Wallis test with Dunn's correction). (i) Input resistance decreased in iSPNs (control: $48.22 \mathrm{M} \Omega, n=37$; haloperidol: $4 \mathrm{I} .53 \mathrm{M} \Omega, n=34$; MannWhitney test, $p=0.02)$. (j, $k, 1)$ Action potential (AP) threshold, resting membrane potential (RMP), and AP peak voltage were all unchanged in iSPNs following haloperidol (control AP threshold: $-41.49 \mathrm{mV}, n=37$; haloperidol AP threshold: $-40.46 \mathrm{mV}, n=34$; Mann-Whitney test, $p=0.17$; control RMP: - 85.99 mV, $n=37$; haloperidol RMP: $-86.27 \mathrm{mV}, n=34$; Mann-Whitney test, $p=0.48$; control AP peak: $43.49 \mathrm{mV}, n=37$; haloperidol AP peak: $44.82 \mathrm{mV}$, $n=34$; Mann-Whitney test, $p=0.63$ ). Asterisks indicate $p<0.05$. 


\section{Dendritic Reconstruction}

For anatomical reconstructions, $0.2 \%$ biocytin was included in the recording solution and allowed to dialyze into the cell for at least $30 \mathrm{~min}$ during whole-cell patch-clamp. Slices were recovered and fixed in $4 \%$ paraformaldehyde for $12 \mathrm{~h}$. Following 4 washes with phosphate-buffered saline (PBS), fixed slices were reacted with $2 \mu \mathrm{g} / \mathrm{ml}$ streptavidinAlexaFluor 594 (Thermo Fisher Scientific, Waltham, MA) in PBS with $1 \%$ normal goat serum, $2 \%$ Triton-X for $12-18 \mathrm{~h}$ on a reciprocal shaker. Slices were washed 4 times with PBS and mounted on slides using ProLong Gold (Thermo Fisher Scientific). Slides were visualized on a 2PLSM setup using a $60 \mathrm{X} / 1.2 \mathrm{NA}$ water immersion objective (Olympus). For whole-cell reconstructions, $0.5 \mu \mathrm{m}$ serial optical sections (Z-stacks) were obtained with $0.386 \mu \mathrm{m}^{2}$ pixel resolution. Four $Z$-stacks per cell were stitched together using volume integration and alignment software (VIAS, Mt Sinai Computational Neurobiology and Imaging Center), and subsequently reconstructed and analyzed using Neurolucida (MicroBrightField, Williston, VT). For spine reconstructions, $0.15 \mu \mathrm{m} Z$-stacks with $0.132 \mu \mathrm{m}^{2}$ pixels were obtained from 2 proximal and 2 distal sections per cell. Z-stacks were deconvolved using AutoQuant (MediaCybernetics, Rockville, $\mathrm{MD}$ ) and spines were counted in three dimensions semiautomatically using NeuronStudio (CNIC, Mount Sinai School of Medicine, New York, NY).

\section{Gene Expression Profiling}

Quantitative polymerase chain reaction (qPCR) was used as previously described (Chan et al, 2012; Plotkin et al, 2013, 2014) to determine the changes in transcript expression following haloperidol administration. In brief, the striatum from haloperidol- or control-treated animals was microdissected and iSPNs were separated out via fluorescenceassisted cell sorting (FACS) based on their eGFP expression. Total mRNA was isolated with the RNeasy Micro Kit (Qiagen, Valencia, CA) before synthesizing cDNA using qScript cDNA Supermix (Quanta Biosciences, Gaithersburg, MD). Real-time PCR was performed using Fast SYBR Mastermix (Applied Biosystems, Waltham, MA) on a StepOnePlus thermocycler (Applied Biosystems). The thermal cycling protocol began with a denaturing step at $95^{\circ} \mathrm{C}$ for $20 \mathrm{~s}$ and then completed 40 cycles of $95^{\circ} \mathrm{C}$ for $3 \mathrm{~s}$ and $60^{\circ}$ $\mathrm{C}$ for $30 \mathrm{~s}$. The PCR cycle threshold $\left(\mathrm{C}_{\mathrm{T}}\right)$ values were measured during the exponential phase of the $\mathrm{PCR}$ reaction and a comparative quantification method $\left(\Delta \Delta \mathrm{C}_{\mathrm{T}}\right)$ was used to identify differences in gene expression level. An ensemble reference was calculated from the stability-based weighted $\mathrm{C}_{\mathrm{T}} \mathrm{s}$ of a panel of reference genes (Gapdh, Atp5b, Cyc1, Eif4az, Gusb, Hmbs, Actb, Uchl1). Experiments for each gene of interest were run in triplicate to generate medians for comparison.

\section{Data Analysis and Statistics}

Data were analyzed using Clampfit 10 (Molecular Devices), Matlab (Mathworks, Natick, MA), and MiniAnalysis (Synaptosoft, Decatur, GA). Statistics were performed in Prism 6 (GraphPad, La Jolla, CA). Data are plotted as mean \pm SEM and summary statistics are presented in box- and-whisker plots showing median, quartiles, and 10-90\% range. Statistical analysis was performed using the nonparametric Mann-Whitney $U$ and Kruskal-Wallis (with Dunn's multiple comparison post hoc) tests of significance. Data are considered significant when $p<0.05$.

\section{RESULTS}

\section{Haloperidol Diminished Intrinsic Excitability of iSPNs, But Not dSPNs}

To investigate the effect of long-term antipsychotic administration on the striatum, BAC transgenic animals were used to allow the unambiguous identification of dSPNs and iSPNs in the slice via somatic eGFP expression using epifluorescence microscopy (Gertler et al, 2008). BAC mice were injected with the typical antipsychotic haloperidol or saline for 2 weeks and then whole-cell patch-clamp recordings were performed in ex vivo striatal slices. Intrinsic excitability was assessed by graded current injection in current-clamp mode. Haloperidol treatment had no effect on dSPN intrinsic excitability (Figures 1d, f and h). However, haloperidol treatment decreased the excitability of iSPNs (Figures 1e and g), a change reflected in a greater rheobase current (the amount of current needed to produce at least one action potential, assessed in $20 \mathrm{pA}$ increments) (Figure $1 \mathrm{~h}$ ).

To ensure that the effect seen in iSPNs was a long-term adaptation, rather than an acute effect of haloperidol, previously untreated mice were injected with a single dose of haloperidol and recorded from $24 \mathrm{~h}$ later. In this case, there were no differences found in the intrinsic excitability of iSPNs (Supplementary Figure S1). To further characterize the time course of the effect, rheobase was measured in iSPNs from mice treated for 5 days (rather than 14); at this time point, rheobase was unchanged (Figure 1h). To determine whether the effect was specific to haloperidol, the typical antipsychotic perphenazine was administered. Perphenazine is a medium-potency phenothiazine antipsychotic that antagonizes $D_{2}$ receptors. Fourteen days of perphenazine administration had a similar effect on rheobase as 14 days of haloperidol (Figure $1 \mathrm{~h}$ ).

Work in rats has suggested that administration of antipsychotics with osmotic mini-pumps rather than IP injections may more accurately mimic human dosing, as assessed by in vivo $\mathrm{D}_{2} \mathrm{R}$ occupancy (Kapur et al, 2003). For this reason, the 2-week administration was repeated using osmotic mini-pumps to deliver haloperidol $(0.25 \mathrm{mg} / \mathrm{kg}$ per day). The results using this delivery method reproduced the drop in intrinsic excitability found using IP injections (Supplementary Figure S2).

To better understand the mechanism underlying the change in excitability seen in iSPNs, input resistance, action potential (AP) threshold, resting membrane potential (RMP), and peak AP voltage were measured. The input resistance was calculated from the slope of the best-fit line of the steady-state voltages obtained following a series of hyperpolarizing somatic current injections $(-150$ to $-70 \mathrm{pA}, 20 \mathrm{pA}$ intervals). Using this method, the input resistance for iSPNs in haloperidol-treated animals was significantly less than that of controls, but the AP threshold, RMP, and AP peak were all unchanged (Figures 1i-l). 


\section{Kir2 $\mathrm{K}^{+}$Channel Currents Were Increased by Haloperidol Treatment}

The Kir2 family of inward rectifying $\mathrm{K}^{+}$channels are major determinants of the resting conductance and basal excitability of SPNs (Mermelstein et al, 1998; Nisenbaum and Wilson, 1995; Uchimura et al, 1989). To determine whether Kir2 $\mathrm{K}^{+}$channel expression was altered by haloperidol treatment, iSPNs from treated and control mice were isolated using FACS and then qPCR was performed (Chan et al, 2012; Plotkin et al, 2013, 2014). The abundance of Kir2.3 mRNA was increased by haloperidol treatment, but Kir2.1, 2.2, and 2.4 mRNAs were unchanged (Figure 2a).

Voltage-clamp recordings of iSPNs from haloperidol- or vehicle-treated animals were performed to see whether Kir2.3 mRNA upregulation resulted in increased protein and current. Voltage-current plots from control- and haloperidol treated animals revealed a divergence around the $\mathrm{K}^{+}$equilibrium potential, suggesting that Kir channel currents were increased by haloperidol treatment (Figure $2 \mathrm{~b}$ ). To provide an additional test of this hypothesis, somatic voltage ramps ( -150 to $-30 \mathrm{mV}, 200 \mathrm{~ms}$ ) were performed and difference currents were computed; these difference currents had a reversal near the $\mathrm{K}^{+}$equilibrium potential and demonstrated inward rectification, both typical features of Kir2 channels (Figure 2c). Finally, iSPNs were voltage-clamped at $-60 \mathrm{mV}$ (inactivating $\mathrm{Kv} 1$ and $\mathrm{Kv} 4$ channels but leaving Kir2 channels open) and then stepped to $-130 \mathrm{mV}$; control- and haloperidol-treated iSPNs revealed a difference in the maximum current (Figures $2 \mathrm{~d}$ and e). These results are all consistent with the hypothesis that the upregulation of Kir2.3 mRNA found in haloperidol-treated animals resulted in increased Kir2 channel currents and reduced intrinsic excitability of iSPNs.

It is possible that other mechanisms also contribute to the drop in excitability seen following chronic haloperidol treatment. Lesioning dopaminergic neurons caused a shrinkage of SPN dendrites (Fieblinger et al, 2014) and $\mathrm{D}_{2} \mathrm{R}$ overexpression resulted in an expanded dendritic arbor (Cazorla et al, 2012). To determine whether there were dendritic changes following haloperidol treatment, iSPNs from treated and control animals were reconstructed and Sholl analysis was performed (Supplementary Figures S3A-C). The total dendritic length of iSPNs was unchanged by haloperidol treatment (Supplementary Figure S3D). Proximal dendritic morphology has a greater impact on somatically assessed excitability than distal morphology, and hence remodeling without a change in the total length could still conceivably affect excitability. However, haloperidol treatment did not change the number of primary dendrites, nodes, or terminal dendritic branches (Supplementary Figures S3E-G). Thus, dendritic remodeling does not appear to contribute to the drop in excitability seen in iSPNs following chronic haloperidol exposure.

\section{Haloperidol Decreased Spine Density}

Loss of dopaminergic signaling has been shown to cause a selective reduction in iSPN spine density (Day et al, 2006; Fieblinger et al, 2014). Studies of chronic haloperidol administration show less consensus, with some reporting decreased spine density in SPNs (Kelley et al, 1997; Roberts
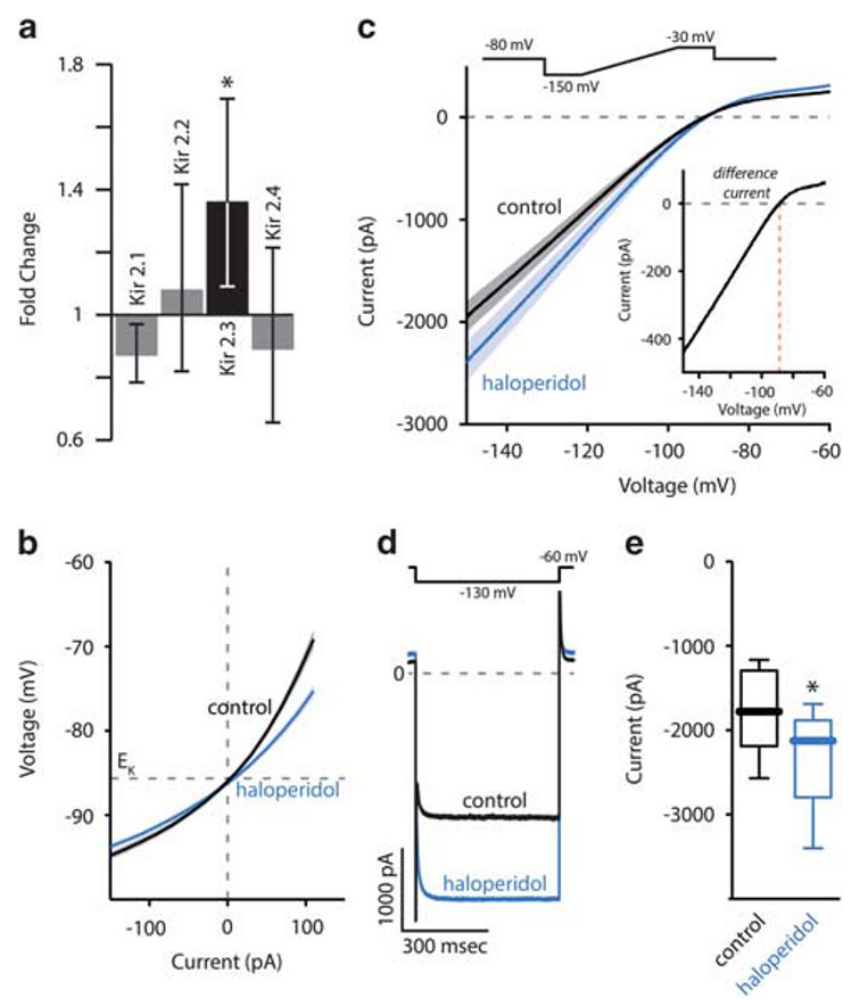

Figure 2 Haloperidol treatment caused Kir2.3 upregulation and increased Kir2-mediated currents. (a) qPCR was performed on iSPNs isolated via their eGFP expression using fluorescence-activated cell sorting. Data are presented as fold change ( $F C$ ) calculated from $\Delta \Delta C_{T}$ values. Only Kir2.3 was significantly increased in the haloperidol group (Kir2.I FC: 0.87, $p=0.33$; Kir2.2 FC: I.08, $p=0.36$; Kir2.3 FC: 1.36, $p=0.00$ I; Kir2.4 FC: 0.89, $p=0.80$; control $n=13$, haloperidol $n=13$; Mann-Whitney test). Dark shading indicates significance. (b) Voltage-current plot determined from current steps in voltage-clamp mode. iSPNs were recorded in control (black) and haloperidol-treated (blue) animals. Shading indicates SEM. (c) Mean current response to a $200 \mathrm{~ms}$ voltage ramp (from - 150 to $-30 \mathrm{mV}$, inset, top). The difference current is also shown (inset, right). (d, e) Haloperidol increased the peak inward current measured by stepping cells from a holding potential of -60 to $-130 \mathrm{mV}$ (control: $-1779 \mathrm{pA}, n=9$; haloperidol $-2126 \mathrm{pA}, n=9$; Mann-Whitney test, $p=0.03$ ). Asterisk indicates $p<0.05$.

et al, 1995), and others increased spine density (Kerns et al, 1992). However, none of the haloperidol studies distinguished between iSPNs and dSPNs that would be expected to have a major impact on the outcome. Using BAC transgenic mice, identified iSPNs were filled with biocytin using a patch electrode, fixed, optically sectioned using 2PLSM, and then reconstructed in 3D using standard approaches (Dumitriu et al, 2011; Gertler et al, 2008). Both proximal (centered at $50 \mu \mathrm{m}$ from the soma) and distal (centered at $110 \mu \mathrm{m}$ ) segments of dendrite were examined and estimates of spine density were generated (Figures $3 \mathrm{a}$ and $\mathrm{b}$ ). Haloperidol administration decreased the median spine density of both proximal and distal iSPN dendrites; this drop was evident at 5 days of treatment and persisted through 14 days of treatment (Figure 3c). Although median spine densities were similar at proximal and distal locations, the variability of the measurements was considerably less in distal dendrites, suggesting the effect was more robust. Perphenazine treatment (14 days) also was effective in decreasing spine density of iSPNs (Figure 3c). 
a

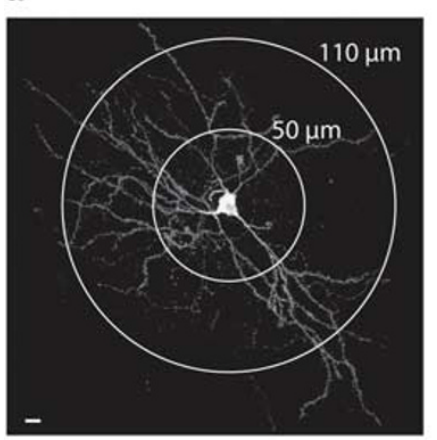

d

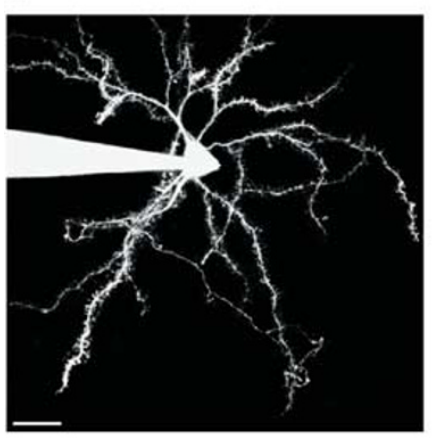

b

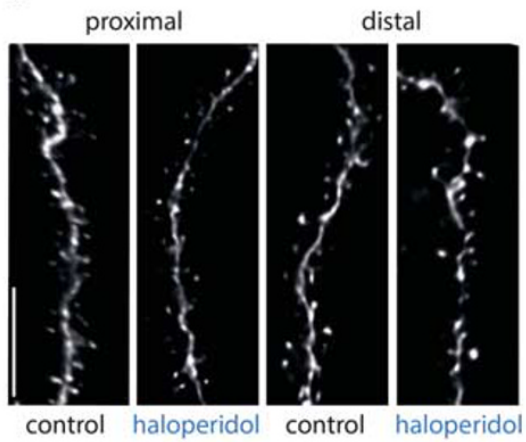

C

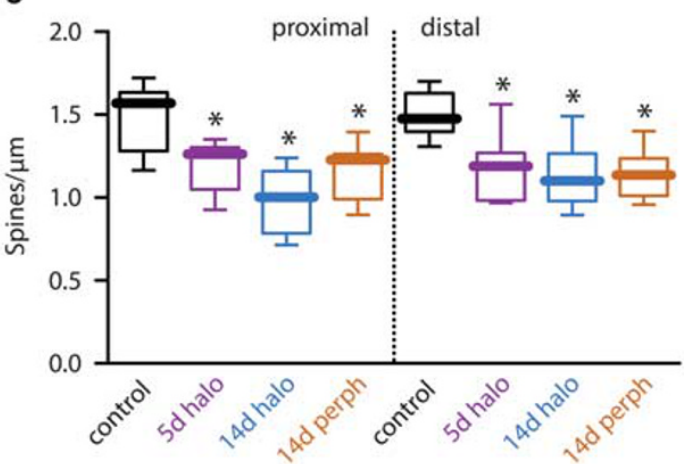

f

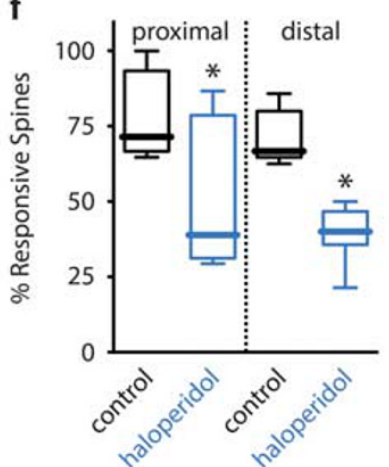

Figure 3 Haloperidol induced the loss of cortically responsive spines. (a) A maximum intensity projection (MIP) of a biocytin-stained iSPN from a vehicleinjected $\mathrm{D}_{2}$ eGFP animal. Concentric circles indicate the proximal ( $50 \mu \mathrm{m}$ from the soma) and distal distances (>90 $\mu \mathrm{m}$ from the soma) for spine counting and subcellular channelrhodopsin-assisted circuit mapping (sCRACM). (b) Representative deconvolved MIPs of dendritic segments used for spine counting. (c) Spine density decreased in both proximal and distal regions with 5 days (5d) of haloperidol (proximal: control=1.56 spines/ $/ \mathrm{mm}, n=8$; $5 \mathrm{~d}$ haloperidol $=1.26$ spines $/ \mu \mathrm{m}, n=15, p=0.05$; distal: control $=1.48$ spines $/ \mu \mathrm{m}, n=8 ; 5 \mathrm{~d}$ haloperidol $=1.19$ spines $/ \mu \mathrm{m}, n=15, p=0.004, \mathrm{Kruskal}-\mathrm{Wallis}$ test with Dunn's correction), with I4 days ( $4 \mathrm{dd}$ ) of haloperidol (proximal: control $=1.56$ spines $/ \mathrm{um}, \mid 4 \mathrm{~d}$ haloperidol $=1.00 \mathrm{spines} / \mathrm{\mu m}$, $p=0.00 \mathrm{I}$; distal: control = I.48 spines/ $\mathrm{mm}, 14 \mathrm{~d}$ haloperidol $=1.10$ spines/ $\mathrm{mm}, p=0.008$; control $n=8,14 \mathrm{~d}$ haloperidol $n=6$, Kruskal-Wallis test with Dunn's correction), and

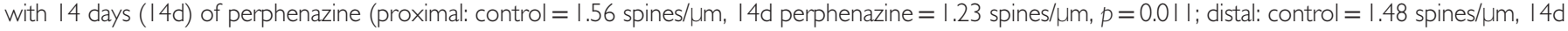
perphenazine $=1.14$ spines $/ \mu m, p<0.001$; control $n=8$, I4d perphenazine $n=16$, Kruskal-Wallis test with Dunn's correction). (d) Sample 2PLSM MIP of an iSPN (left) that was patch-clamped and dialyzed with Alexa Fluor 568 and a region of dendrite (right) targeted for sCRACM. Red circles indicate regions sampled for focal blue laser stimulation of ChR2. (e) A cartoon representation (left) of the region of dendrite shown in (d). Somatic voltage records (right) in response to focal laser stimulation; when laser stimulation produced a response the spines were classified as cortically responsive. (f) Haloperidol treatment reduced the percent of cortical-responsive spines in both regions examined (proximal: control $=71.43 \%$, haloperidol $=38.89 \%, p=0.038$; distal: control $=66.67 \%$, haloperidol $=40.00, p=0.0006$; control $n=7$, haloperidol $n=7$, Mann-Whitney test). Scale bars, $10 \mu m$; asterisks indicate $p<0.05$.

SPNs receive excitatory glutamatergic innervation from both the cerebral cortex and thalamus. However, the majority of axospinous synapses are cortical in origin (Smith et al, 2004). To determine whether cortical axospinous synapses were being lost following haloperidol treatment, the sCRACM approach was used (Fieblinger et al, 2014; Petreanu et al, 2009). This method uses focal illumination of spine heads imaged using 2PLSM in ex vivo brain slices. When illuminated, presynaptic terminals expressing channelrhodopsin (ChR2) depolarize and release neurotransmitter, generating a postsynaptic current detected by a somatic patch electrode. By expressing ChR2 in presynaptic terminals of a given type, the terminals can be mapped. To map corticostriatal synapses onto iSPNs, Thy1ChR2 mice that express channelrhodopsin in corticostriatal but not thalamostriatal neurons were crossed with $\mathrm{D}_{2}$ eGFP mice (Plotkin et al, 2014). These mice were treated with haloperidol or vehicle control. After treatment, brain slices were prepared and iSPNs were patched and filled with an Alexa dye to allow the visualization of dendrites. Spines were then interrogated with a blue laser to determine whether a cortical terminal was present (Figures $3 \mathrm{~d}$ and e). In control iSPNs, roughly $70 \%$ of the spines on proximal and distal dendrites had detectable cortical synapses; this is in good agreement with previous results using this approach and anatomical estimates of corticostriatal synapse density (Fieblinger et al, 2014; Plotkin et al, 2014; Smith et al, 2009; Villalba and Smith, 2011). After haloperidol treatment, this percentage dropped to $\sim 40 \%$ in both proximal and distal dendrites (Figure 3f). These results suggest that there was a selective pruning of cortical synapses by haloperidol treatment.

\section{Haloperidol Increased the Strength of Remaining Axospinous Synapses}

Activation of $\mathrm{D}_{2}$ Rs is necessary for endocannabinoid (eCB)mediated long-term synaptic depression (LTD) in the striatum (Gerfen and Surmeier, 2011; Kreitzer and Malenka, 2007). In this form of plasticity, retrograde signaling of eCBs activates presynaptic cannabinoid 1 receptors that decrease release probability. Chronic blockade 
of $\mathrm{D}_{2} \mathrm{Rs}$ by haloperidol might blunt normal eCB-LTD induction, leading to de-depression of corticostriatal synapses. To assess this possibility, the paired-pulse ratio (PPR), which can indicate changes in release probability (Zucker and Regehr, 2002), was measured in iSPNs in response to electrical stimulation of corticostriatal fibers (Figure 4a). As would be expected from the loss of eCB-LTD, the PPR in iSPNs was significantly lower in haloperidoltreated animals than in vehicle controls (Figure 4b), indicating an increased probability of release.

To determine whether synaptic strength was affected by haloperidol treatment, asynchronous corticostriatal mEPSCs were evoked by electrical stimulation of corticostriatal axons in aCSF containing $\mathrm{Sr}^{2+}$ in place of $\mathrm{Ca}^{2+}$ (Ding et al, 2008) (Figures $4 \mathrm{c}$ and $\mathrm{d}$ ); $\mathrm{Sr}^{2+}$ replacement increases asynchronous quantal release, allowing mEPSCs of known presynaptic origin to be determined (Choi and Lovinger, 1997). Although the frequency of asynchronous mEPSCs is not readily related to release probability, the amplitude of mEPSCs provides a direct measure of synaptic strength. Because postsynaptic glutamate receptors at this synapse are not saturated normally (Higley et al, 2009), an elevation in presynaptic release probability could result in an increase in mEPSC amplitude. Indeed, the average corticostriatal mEPSC amplitude in iSPNs was increased by haloperidol treatment, suggesting a loss of presynaptic eCB-LTD (Figures $4 \mathrm{e}$ and f; Supplementary Figure S4). In addition, analysis of corticostriatal synaptic strength obtained via optogenetic stimulation in Thy1-ChR2 mice revealed an increase in the median amplitude of response following haloperidol treatment (control: $4.3 \mathrm{pA}, n=134$ spines; haloperidol: $5.3 \mathrm{pA}, n=95$ spines; Mann-Whitney U-test; $p=0.004)$ that was roughly equivalent $(\sim 25 \%)$ to that seen with $\mathrm{Sr}^{2+}$ mEPSCs.

Finally, the ratio of $N$-methyl-D-aspartate receptor (NMDAR) currents to $\alpha$-amino-3-hydroxy-5-methyl-4-isoxazolepropionic acid receptor (AMPAR) currents at corticostriatal synapses was measured using an established technique (Myme et al, 2003) (Supplementary Figure S5A). This ratio is often used as a means of determining whether postsynaptic long-term depression or potentiation, which changes the relative abundance of synaptic AMPARs, has occurred (Yang and Calakos, 2013). The time course of AMPAR currents was first determined at $-80 \mathrm{mV}$, a potential at which $\mathrm{Mg}^{2+}$ blocks NMDAR currents. Then the cortex was stimulated while holding the cell at $+40 \mathrm{mV}$ to reveal NMDAR currents in addition to AMPAR currents. The AMPAR component was taken at the time of peak AMPAR current assessed at $-80 \mathrm{mV}$, whereas the NMDAR component was taken 40 ms later, after the AMPAR current had completely decayed (Supplementary Figure S5A). Using this technique, haloperidol treatment did not change the synaptic NMDA/AMPA current ratio (Supplementary Figure S5B), suggesting a presynaptic mechanism of action.

\section{Haloperidol Tolerance Paralleled Adaptations in iSPNs}

Catalepsy is a basal ganglia-mediated behavior that can be triggered by antipsychotic administration (Bateup et al, 2010). Tolerance to the cataleptic effects of haloperidol develops with repeated treatment (Ezrin-Waters and Seeman, 1977). To determine whether the development of tolerance paralleled the adaptations in iSPNs, mice were a
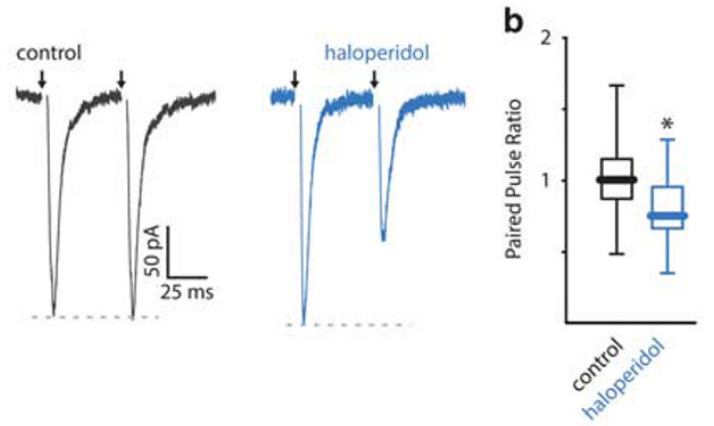

c

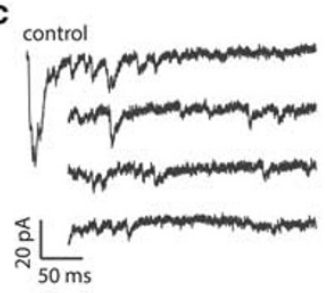

d
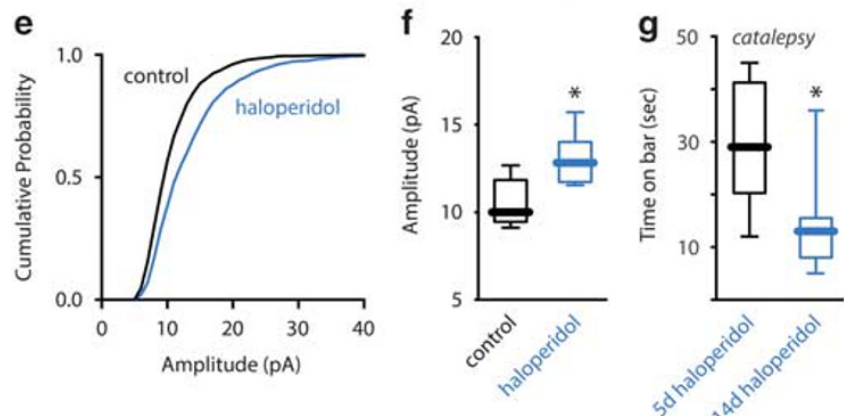

Figure 4 Haloperidol strengthened the remaining corticostriatal synapses. (a) Representative EPSCs evoked by paired-pulse $(20 \mathrm{~Hz})$ electrical stimulation of the cortex. Arrows denote cortical stimuli. (b) The PPR in controls was mildly facilitating but was depressing in haloperidol animals, indicating an increased probability of release (control: I.00, $n=25$; haloperidol: 0.75, $n=19$; Mann-Whitney test, $p=0.007$ ). Representative traces showing asynchronous release evoked by cortical stimulation measured in iSPNs of control (c) and haloperidol-treated (d) animals. (e) Cumulative probability distribution of mEPSC amplitude. ( $f$ ) Haloperidol increased mEPSC amplitude as compared with control (control: 10.0I pA, $n=8$; haloperidol: I2.83 pA, $n=7$; Mann-Whitney test, $p=0.004$ ). (g) Mice developed tolerance to catalepsy following 14 days of haloperidol administration (time on bar: $5 \mathrm{~d}$ haloperidol: $29 \mathrm{~s}, p=8$; $14 \mathrm{~d}$ haloperidol: $13 \mathrm{~s}, n=9$; Mann-Whitney test, $p=0.007$ ). Asterisks indicate $p<0.05$.

treated for 5 or 14 days with haloperidol and their tolerance to catalepsy measured using a standard test (Peterson et al, 2012). The cataleptic tolerance to haloperidol was progressive, increasing significantly from 5 to 14 days of treatment (Figure 4g), paralleling the changes in intrinsic excitability of iSPNs.

\section{DISCUSSION}

This study shows that chronic (2 weeks) treatment of mice with haloperidol induces a concerted set of adaptations specifically in striatal iSPNs. Although haloperidol is a nonselective, albeit preferential, $\mathrm{D}_{2}$ receptor antagonist (Bymaster et al, 1996), both the downregulation of intrinsic excitability and of excitatory synaptic connectivity with 
cortical pyramidal neurons appear to reflect a form of homeostatic plasticity triggered by the loss of tonic, inhibitory $\mathrm{D}_{2} \mathrm{R}$-mediated modulation of iSPNs. These homeostatic adaptations should serve to normalize the average spiking rate of iSPNs following neuroleptic treatment, diminishing the propensity of this network to indiscriminately suppress goal-directed movement and habits. However, in parallel, neuroleptic treatment led to the strengthening of a subset of corticostriatal synapses, increasing their ability to drive iSPN activity. Thus, chronic neuroleptic treatment leads to a reorganization of the corticostriatal circuitry controlling active suppression of thoughts and movements. Whereas the changes in synaptic density occurred rapidly, alterations in intrinsic excitability were slower. Although our behavioral data indicate that the changes in intrinsic excitability parallels tolerance to catalepsy, the time course of these adaptations also mirrors the delay between neuroleptic administration and therapeutic benefit; further study in a rodent model of schizophrenia would be of value to determine whether the reported iSPN adaptations are also associated with symptoms of schizophrenia, particularly positive symptoms.

\section{Homeostatic Adaptations to Chronic Haloperidol}

$\mathrm{D}_{2} \mathrm{R}$ signaling has both rapid and slow neuromodulatory effects on iSPNs. The rapid neuromodulatory effects of D2R signaling are mediated by $\mathrm{G}_{\mathrm{i} / \mathrm{o}}$ proteins coupled to adenylyl cyclase and phospholipase $\mathrm{C}$, leading to altered gating of $\mathrm{Na}^{+}, \mathrm{Ca}^{2+}$, and $\mathrm{K}^{+}$channels (Gerfen and Surmeier, 2011). The same signaling pathways mediate slow, lasting downregulation of excitatory synaptic connections (Gerfen and Surmeier, 2011; Kreitzer and Malenka, 2007). Thus, both slow and fast effects of $\mathrm{D}_{2} \mathrm{R}$ signaling tend to decrease the activity of iSPNs. How then does chronic haloperidol treatment, which should decrease inhibitory $\mathrm{D}_{2} \mathrm{R}$ signaling, lead to a drop in iSPN excitability? By attenuating tonic inhibitory $\mathrm{D}_{2} \mathrm{R}$ signaling in iSPNs, haloperidol should increase their spiking rate (West and Grace, 2002; Zhang and Linden, 2003). With high doses of haloperidol, this disinhibition of iSPNs broadly suppresses movement, inducing catalepsy. However, sustained deviations of spike rate away from a neuron's predetermined set-point induces homeostatic compensation or plasticity (Marder and Goaillard, 2006; Turrigiano, 2008). Two forms of homeostatic plasticity have been described: intrinsic and synaptic. Intrinsic homeostatic plasticity leads to alterations in postsynaptic ion channel expression and function that control spike generation. Synaptic homeostatic plasticity or scaling leads to changes in the strength of excitatory glutamatergic synapses that drive neurons to spike.

In the case of iSPNs, sustained antagonism of inhibitory $\mathrm{D}_{2} \mathrm{R}$ signaling should engage a negative form of homeostatic plasticity that lowers, and hence normalizes, spiking rate. Indeed this is what was found. In iSPNs from mice treated with haloperidol, the ability to generate spikes from intrasomatic current injection was decreased and change paralleled the development of tolerance to catalepsy. This drop in excitability was attributable in part to an upregulation in the expression and function of Kir2.3 $\mathrm{K}^{+}$channels. Both mRNA for this key subunit of Kir2 channels and Kir2 channel currents were upregulated by haloperidol treatment.
This observation is consistent with recent work showing that upregulation of $\mathrm{D}_{2} \mathrm{R}$ expression increased SPN excitability by downregulating Kir2 channel function (Cazorla et al, 2012).

In addition to intrinsic homeostatic plasticity, iSPNs from haloperidol-treated mice also pruned excitatory, glutamatergic axospinous synapses on both proximal and distal dendrites that can be interpreted as a form of synaptic homeostatic plasticity. This pruning was similar to that seen following striatal dopamine depletion (Day et al, 2006; Fieblinger et al, 2014). The pruning induced by haloperidol treatment appeared to occur primarily at corticostriatal, axospinous synapses, like that seen following dopamine depletion. However, rather than all excitatory synapses simply being downregulated (scaling) to maintain relative synaptic strength and the information embedded in these weights (Turrigiano, 2008), a subset of axospinous synapses were eliminated and others strengthened, as judged by the increase in $\mathrm{Sr}^{2+}$ mEPSC amplitude following haloperidol treatment. The strengthening of these synapses is attributable to dedepression following the loss of $\mathrm{D}_{2} \mathrm{R}$-dependent, presynaptic LTD (Gerfen and Surmeier, 2011). Consistent with a presynaptic locus for this change, the NMDAR/ AMPAR current ratio-a commonly used measure of postsynaptic plasticity-was unaltered at corticostriatal synapses following haloperidol treatment. Moreover, the PPR at this synapse decreased, suggesting an increase in glutamate release probability, as expected following a loss of presynaptic LTD. This inference is consistent with ultrastructural studies showing that chronic haloperidol treatment increases the number of perforated striatal synapses (Meshul and Casey, 1989; Meshul et al, 1996), providing a structural foundation for our physiological observations (Calverley and Jones, 1990; Higley et al, 2009).

It remains to be seen how SPN homeostatic plasticity is regulated, but one potential mechanism is through Cav1 (L-type) $\mathrm{Ca}^{2+}$ channels that are robustly expressed by SPNs. Several studies have implicated Cav1 channels in the regulation of homeostatic plasticity (Turrigiano, 2008). This possibility is of considerable interest, as genome-wide association studies have implicated Cav1 polymorphisms in psychiatric diseases, particularly schizophrenia (Cross-Disorder Group of the Psychiatric Genomics Consortium, 2013).

\section{Do Cholinergic Interneurons Contribute to the Effects of Haloperidol?}

$\mathrm{D}_{2} \mathrm{R}$ expression is not limited to iSPNs in the striatum. It is possible that these other cell types shape the iSPN adaptations to chronic haloperidol. The most probable participant in the response to haloperidol are $\mathrm{D}_{2} \mathrm{R}$ expressing cholinergic interneurons (ChIs). $\mathrm{D}_{2} \mathrm{R}$ signaling in these neurons suppresses their ongoing pacemaking activity and acetylcholine release (Aosaki et al, 1994; Ding et al, 2010; Maurice et al, 2004; Stoof and Kebabian, 1982). Acetylcholine has a variety of striatal effects, but the most relevant in this context is the elevation in iSPN intrinsic excitability mediated by suppression of Kv7 (KCNQ) and Kir2 $\mathrm{K}^{+}$channels (Galarraga et al, 1999; Shen et al, 2005, 2007). It is possible that neuroleptic-induced elevation in cholinergic signaling contributes to the observed adaptations in iSPNs (Shen et al, 2007). However, in vivo microdialysis in 
rodents chronically treated with haloperidol have found no change in basal ACh tone (Osborne et al, 1994).

\section{Implications for Schizophrenia}

Typical neuroleptics are most effective in reducing the positive symptoms of schizophrenia. These symptoms are thought to arise from hyperactivity of $\mathrm{D}_{2} \mathrm{R}$ signaling and hypoexcitability of the indirect pathway-a key part of the brain circuitry that suppresses contextually inappropriate ideation, habits, and goal-directed activity. In principle, neuroleptics should immediately alleviate these symptoms if this simple model is correct. However, they do not, suggesting that there is something else awry in the schizophrenic brain. What this model lacks is an appreciation for the homeostatic potential of iSPNs. Cazorla et al (2012) show that sustained upregulation in D2R signaling, as might be found in schizophrenia, induces homeostatic alterations in the Kir2-dependent integrative mechanisms of SPNs. Our results suggest that haloperidol, and other typical antipsychotics such as perphenazine, can reverse these Kir2-dependent adaptations, but this reversal takes days or weeks.

In addition, there are compelling reasons to think that synaptic dysfunction contributes to the schizophrenic phenotype (Faludi and Mirnics, 2011; Mirnics et al, 2006). In agreement with this view, long-term antipsychotic treatment remodels axospinous machinery controlling corticostriatal synaptic function (Critchlow et al, 2006; Faludi and Mirnics, 2011; Lidow et al, 2001). Our results add an important dimension to this literature, showing that haloperidol slowly remodels corticostriatal circuitry controlling iSPNs, eliminating many synapses but strengthening others.

What remains to be determined are the types of corticostriatal synapses pruned and the types strengthened by haloperidol treatment. These might arise from different cortical regions or circuits. Our current hypothesis is that the axospinous synapses capable of undergoing synaptic plasticity (LTP) are selectively preserved. This would mean that the pruned synapses are not as sensitive to the consequences of choice that is signaled to the striatum by the dopaminergic neurons of the substantia nigra. By preferentially eliminating these synapses, haloperidol might force the activity of iSPNs to be more effectively governed by cortical inputs shaped by external events.

\section{FUNDING AND DISCLOSURE}

The authors declare no conflict of interest.

\section{ACKNOWLEDGMENTS}

We thank Dr Tracy Gertler for her contribution to the early stages of this project, Alexandria Melendez for auxiliary experiments, and Sasha Ulrich for technical assistance. This work was supported by NIMH P50 MH090963, NS34696, NS084735, and the JPB Foundation.

\section{REFERENCES}

Agid O, Kapur S, Arenovich T, Zipursky RB (2003). Delayed-onset hypothesis of antipsychotic action: a hypothesis tested and rejected. Arch Gen Psychiatry 60: 1228-1235.

Aosaki T, Graybiel AM, Kimura M (1994). Effect of the nigrostriatal dopamine system on acquired neural responses in the striatum of behaving monkeys. Science 265: 412-415.

Bateup HS, Santini E, Shen W, Birnbaum S, Valjent E, Surmeier DJ et al (2010). Distinct subclasses of medium spiny neurons differentially regulate striatal motor behaviors. Proc Natl Acad Sci USA 107: 14845-14850.

Bymaster FP, Calligaro DO, Falcone JF, Marsh RD, Moore NA, Tye NC et al (1996). Radioreceptor binding profile of the atypical antipsychotic olanzapine. Neuropsychopharmacology 14: 87-96.

Calverley RK, Jones DG (1990). Contributions of dendritic spines and perforated synapses to synaptic plasticity. Brain Res Brain Res Rev 15: 215-249.

Cazorla M, Shegda M, Ramesh B, Harrison NL, Kellendonk C (2012). Striatal D2 receptors regulate dendritic morphology of medium spiny neurons via Kir2 channels. J Neurosci 32: 2398-2409.

Centonze D, Usiello A, Costa C, Picconi B, Erbs E, Bernardi G et al (2004). Chronic haloperidol promotes corticostriatal long-term potentiation by targeting dopamine D2L receptors. J Neurosci 24 : 8214-8222.

Chan CS, Peterson JD, Gertler TS, Glajch KE, Quintana RE, Cui Q et al (2012). Strain-specific regulation of striatal phenotype in Drd2-eGFP BAC transgenic mice. J Neurosci 32: 9124-9132.

Choi S, Lovinger DM (1997). Decreased frequency but not amplitude of quantal synaptic responses associated with expression of corticostriatal long-term depression. J Neurosci 17: 8613-8620.

Creese I, Burt DR, Snyder SH (1976). Dopamine receptor binding predicts clinical and pharmacological potencies of antischizophrenic drugs. Science 192: 481-483.

Critchlow HM, Maycox PR, Skepper JN, Krylova O (2006). Clozapine and haloperidol differentially regulate dendritic spine formation and synaptogenesis in rat hippocampal neurons. Mol Cell Neurosci 32: 356-365.

Cross-Disorder Group of the Psychiatric Genomics Consortium (2013). Identification of risk loci with shared effects on five major psychiatric disorders: a genome-wide analysis. Lancet 381: 1371-1379.

Day M, Wang Z, Ding J, An X, Ingham CA, Shering AF et al (2006). Selective elimination of glutamatergic synapses on striatopallidal neurons in Parkinson disease models. Nat Neurosci 9: 251-259.

Ding J, Peterson JD, Surmeier DJ (2008). Corticostriatal and thalamostriatal synapses have distinctive properties. J Neurosci $\mathbf{2 8}$ : 6483-6492.

Ding JB, Guzman JN, Peterson JD, Goldberg JA, Surmeier DJ (2010). Thalamic gating of corticostriatal signaling by cholinergic interneurons. Neuron 67: 294-307.

Dumitriu D, Rodriguez A, Morrison JH (2011). High-throughput, detailed, cell-specific neuroanatomy of dendritic spines using microinjection and confocal microscopy. Nat Protoc 6: 1391-1411.

Ezrin-Waters C, Seeman P (1977). Tolerance of haloperidol catalepsy. Eur J Pharmacol 41: 321-327.

Faludi G, Mirnics K (2011). Synaptic changes in the brain of subjects with schizophrenia. Int J Dev Neurosci 29: 305-309.

Fieblinger T, Graves SM, Sebel LE, Alcacer C, Plotkin JL, Gertler TS et al (2014). Cell type-specific plasticity of striatal projection neurons in parkinsonism and L-DOPA-induced dyskinesia. Nat Commun 5: 5316. 
Frank MJ (2011). Computational models of motivated action selection in corticostriatal circuits. Curr Opin Neurobiol 21: 381-386.

Galarraga E, Hernández-López S, Reyes A, Miranda I, BermudezRattoni F, Vilchis C et al (1999). Cholinergic modulation of neostriatal output: a functional antagonism between different types of muscarinic receptors. J Neurosci 19: 3629-3638.

Gerfen CR, Surmeier DJ (2011). Modulation of striatal projection systems by dopamine. Annu Rev Neurosci 34: 441-466.

Gertler TS, Chan CS, Surmeier DJ (2008). Dichotomous anatomical properties of adult striatal medium spiny neurons. J Neurosci 28 : 10814-10824.

Higley MJ, Soler-Llavina GJ, Sabatini BL (2009). Cholinergic modulation of multivesicular release regulates striatal synaptic potency and integration. Nat Neurosci 12: 1121-1128.

Ilijic E, Guzman JN, Surmeier DJ (2011). The L-type channel antagonist isradipine is neuroprotective in a mouse model of Parkinson's disease. Neurobiol Dis 43: 364-371.

Kapur S, VanderSpek SC, Brownlee BA, Nobrega JN (2003). Antipsychotic dosing in preclinical models is often unrepresentative of the clinical condition: a suggested solution based on in vivo occupancy. J Pharmacol Exp Ther 305: 625-631.

Kelley JJ, Gao XM, Tamminga CA, Roberts RC (1997). The effect of chronic haloperidol treatment on dendritic spines in the rat striatum. Exp Neurol 146: 471-478.

Kerns JM, Sierens DK, Kao LC, Klawans HL, Carvey PM (1992). Synaptic plasticity in the rat striatum following chronic haloperidol treatment. Clin Neuropharmacol 15: 488-500.

Kirschbaum KM, Henken S, Hiemke C, Schmitt U (2008). Pharmacodynamic consequences of P-glycoprotein-dependent pharmacokinetics of risperidone and haloperidol in mice. Behav Brain Res 188: 298-303.

Konradi C, Heckers S (2001). Antipsychotic drugs and neuroplasticity: insights into the treatment and neurobiology of schizophrenia. Biol Psychiatry 50: 729-742.

Kreitzer AC, Malenka RC (2007). Endocannabinoid-mediated rescue of striatal LTD and motor deficits in Parkinson's disease models. Nature 445: 643-647.

Laruelle M, Kegeles LS, Abi-Dargham A (2003). Glutamate, dopamine, and schizophrenia: from pathophysiology to treatment. Ann NY Acad Sci 1003: 138-158.

Leucht S, Busch R, Hamann J, Kissling W, Kane JM (2005). Earlyonset hypothesis of antipsychotic drug action: a hypothesis tested, confirmed and extended. Biol Psychiatry 57: 1543-1549.

Lidow MS, Song ZM, Castner SA, Allen PB, Greengard P, Goldman-Rakic PS (2001). Antipsychotic treatment induces alterations in dendrite- and spine-associated proteins in dopamine-rich areas of the primate cerebral cortex. Biol Psychiatry 49: 1-12.

Marder E, Goaillard J-M (2006). Variability, compensation and homeostasis in neuron and network function. Nat Rev Neurosci 7: 563-574.

Maurice N, Mercer J, Chan CS, Hernandez-Lopez S, Held J, Tkatch T et al (2004). D2 dopamine receptor-mediated modulation of voltage-dependent $\mathrm{Na}+$ channels reduces autonomous activity in striatal cholinergic interneurons. J Neurosci 24: 10289-10301.

Mermelstein PG, Song WJ, Tkatch T, Yan Z, Surmeier DJ (1998). Inwardly rectifying potassium (IRK) currents are correlated with IRK subunit expression in rat nucleus accumbens medium spiny neurons. J Neurosci 18: 6650-6661.

Meshul CK, Andreassen OA, Allen C, Jørgensen HA (1996). Correlation of vacuous chewing movements with morphological changes in rats following 1-year treatment with haloperidol. Psychopharmacology 125: 238-247.

Meshul CK, Casey DE (1989). Regional, reversible ultrastructural changes in rat brain with chronic neuroleptic treatment. Brain Res 489: 338-346.
Mirnics K, Levitt P, Lewis DA (2006). Critical appraisal of DNA microarrays in psychiatric genomics. Biol Psychiatry 60: $163-176$.

Myme CIO, Sugino K, Turrigiano GG, Nelson SB (2003). The NMDA-to-AMPA ratio at synapses onto layer $2 / 3$ pyramidal neurons is conserved across prefrontal and visual cortices. J Neurophysiol 90: 771-779.

Naundorf B, Wolf F, Volgushev M (2006). Unique features of action potential initiation in cortical neurons. Nature 440: 1060-1063.

Nisenbaum ES, Wilson CJ (1995). Potassium currents responsible for inward and outward rectification in rat neostriatal spiny projection neurons. J Neurosci 15: 4449-4463.

Osborne PG, O'Connor WT, Beck O, Ungerstedt U (1994). Acute versus chronic haloperidol: relationship between tolerance to catalepsy and striatal and accumbens dopamine, GABA and acetylcholine release. Brain Res 634: 20-30.

Peterson JD, Goldberg JA, Surmeier DJ (2012). Adenosine A2a receptor antagonists attenuate striatal adaptations following dopamine depletion. Neurobiol Dis 45: 409-416.

Petreanu L, Mao T, Sternson SM, Svoboda K (2009). The subcellular organization of neocortical excitatory connections. Nature 457: 1142-1145.

Plotkin JL, Day M, Peterson JD, Xie Z, Kress GJ, Rafalovich I et al (2014). Impaired TrkB receptor signaling underlies corticostriatal dysfunction in Huntington's disease. Neuron 83: 178-188.

Plotkin JL, Shen W, Rafalovich I, Sebel LE, Day M, Chan CS et al (2013). Regulation of dendritic calcium release in striatal spiny projection neurons. J Neurophysiol 110: 2325-2336.

Redgrave P, Rodriguez M, Smith Y, Rodriguez-Oroz MC, Lehericy S, Bergman H et al (2010). Goal-directed and habitual control in the basal ganglia: implications for Parkinson's disease. Nat Rev Neurosci 11: 760-772.

Roberts RC, Gaither LA, Gao XM, Kashyap SM, Tamminga CA (1995). Ultrastructural correlates of haloperidol-induced oral dyskinesias in rat striatum. Synapse 20: 234-243.

Seeman P, Lee T, Chau-Wong M, Wong K (1976). Antipsychotic drug doses and neuroleptic/dopamine receptors. Nature 261: 717-719.

Shen W, Hamilton SE, Nathanson NM, Surmeier DJ (2005). Cholinergic suppression of KCNQ channel currents enhances excitability of striatal medium spiny neurons. J Neurosci 25: 7449-7458.

Shen W, Tian X, Day M, Ulrich S, Tkatch T, Nathanson NM et al (2007). Cholinergic modulation of Kir2 channels selectively elevates dendritic excitability in striatopallidal neurons. Nat Neurosci 10: 1458-1466.

Smith Y, Raju D, Nanda B, Pare J-F, Galvan A, Wichmann T (2009). The thalamostriatal systems: anatomical and functional organization in normal and parkinsonian states. Brain Res Bull 78: 60-68.

Smith Y, Raju DV, Pare J-F, Sidibe M (2004). The thalamostriatal system: a highly specific network of the basal ganglia circuitry. Trends Neurosci 27: 520-527.

Stoof JC, Kebabian JW (1982). Independent in vitro regulation by the D-2 dopamine receptor of dopamine-stimulated efflux of cyclic AMP and $\mathrm{K}+$-stimulated release of acetylcholine from rat neostriatum. Brain Res 250: 263-270.

Tauscher J, Jones C, Remington G, Zipursky RB, Kapur S (2002). Significant dissociation of brain and plasma kinetics with antipsychotics. Mol Psychiatry 7: 317-321.

Tepper JM, Sharpe NA, Koós TZ, Trent F (1998). Postnatal development of the rat neostriatum: electrophysiological, light- and electron-microscopic studies. Dev Neurosci 20: 125-145.

Turrigiano GG (2008). The self-tuning neuron: synaptic scaling of excitatory synapses. Cell 135: 422-435. 
Uchimura N, Cherubini E, North RA (1989). Inward rectification in rat nucleus accumbens neurons. J Neurophysiol 62: 1280-1286. Villalba RM, Smith Y (2011). Differential structural plasticity of corticostriatal and thalamostriatal axo-spinous synapses in MPTP-treated Parkinsonian monkeys. J Comp Neurol 519: 989-1005.

West AR, Grace AA (2002). Opposite influences of endogenous dopamine D1 and D2 receptor activation on activity states and electrophysiological properties of striatal neurons: studies combining in vivo intracellular recordings and reverse microdialysis. J Neurosci 22: 294-304.
Winton-Brown TT, Fusar-Poli P, Ungless MA, Howes OD (2014). Dopaminergic basis of salience dysregulation in psychosis. Trends Neurosci 37: 85-94.

Yang Y, Calakos N (2013). Presynaptic long-term plasticity. Front Synaptic Neurosci 5: 8.

Zhang W, Linden DJ (2003). The other side of the engram: experience-driven changes in neuronal intrinsic excitability. Nat Rev Neurosci 4: 885-900.

Zucker RS, Regehr WG (2002). Short-term synaptic plasticity. Annu Rev Physiol 64: 355-405.

Supplementary Information accompanies the paper on the Neuropsychopharmacology website (http://www.nature.com/npp) 